\title{
REPORT
}

\section{Introduction of Japanese Society of Oral Implantology}

\author{
Takayoshi Kawazoe
}

The Board of Directors, Osaka Dental University, Osaka, Japan

Document code: A CLC number: G113 Received Dec.5,2008

In response to the significant advancement of oral implantology around the world, the Japanese Society of Oral Implantology (JSOI) was established in 1972. Its purpose is to promote and improve oral implantology in Japan, and introduce safe and predictable implant treatment methods.

The Japanese Society of Oral Implantology consists of six local branches (Figure 1), which are responsible for organizing academic conferences and training sessions at designated facilities. They also play important roles in communities by enhancing knowledge and skills necessary for oral implant treatment and providing education and training in oral implantology for local dentists.

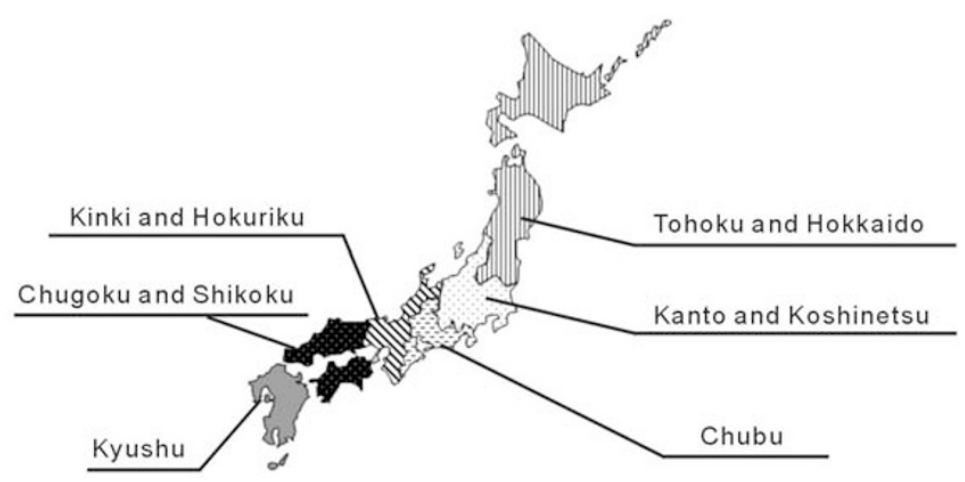

Figure 1 Six branches of JSOI

In 2005, 33 years after its establishment, the Japanese Society of Oral Implantology underwent two major changes: participation in the Japanese Association of Dental Science, and recognition as an incorporated association.

The Japanese Association of Dental Science, an academic association, is a leading dental organization in Japan and a member of The Japan Dental Association. With a membership of about nine million, it includes twenty-one special and nine authorized committees.

The acquisition of the status of an incorporated association was also an important event, which promoted the advancement of the Japanese Society of Oral Implantology. In Japan, there are four types of corporate status approved by the Ministry of Health, Labour and Welfare: incorporated association, incorporated foundation, intermediate corporation, and incorporated nonprofit organization. It is necessary to meet the most demanding requirements to be recognized as an incorporated association.

Now, the Japanese Society of Oral Implantology is highly praised and recognized by society as an organization devoted to promote and improve oral implant treatment and contribute to the welfare of people in Japan.

As the social status of the Japanese Society of Oral Implantology improves and our responsibility increases, so does the number of memberscurrently 9909 (Figure 2).

Over the past few years, in particular, there has 


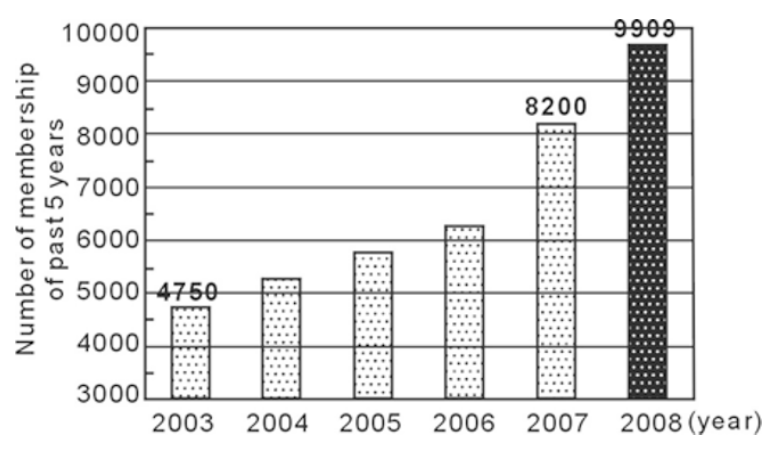

Figure 2 Membership of JSOI in the past 5 years

been a sharp increase in the number of people supporting and joining the Japanese Society of Oral Implantology: an increase of approximately 3800 over the past two years. One of the reasons for this advancement of the Japanese Society of Oral Implantology is assumed to be the "Establishment of a medical specialist system in oral implantology". Members of the Japanese Society of Oral Implantology hope to facilitate specialized implant treatment, and, as certified specialists, provide reliable implant care and respond to the expectations of society.

In Japan, only academic societies that meet the standards set by the Ministry of Health, Labour and Welfare are allowed to certify a person as a specialist. Members in such society should be no less than 1000 and 80 percent of them should be doctors. The Japanese Society of Oral Implantology provides a career path program for those who want to become certified specialists, in which they receive medical training required in each area of medicine for ten years (Figure 3). We perform the thorough screening of candidates for certifying dentists, specialists, and supervisory dentists, including a review of case reports and other achievements, interviews, and written examinations, in accordance with the regulations stipulated by the society.

Oral implant specialists are experts in oral implantology, and include the following certified professionals: "The Japanese Society of Oral Implantology certifying doctor of oral implantology" "Specialist in oral implantology" "Supervisory doctor in oral implantology" "Supervisory doctor in a biological field related to oral implantology" "Dental hygienist specialized in dental implant" and "Dental technician specialized in implant prosthesis". Table 1 shows number of specialist and supervisory doctor in oral implantology.

Setting academic advancement as a goal, we have been conducting intensive studies focused on the following six subjects: "Bone augmentation, induction, and regeneration" "Early loading and immediate loading" "Soft tissue management" "Esthetic recovery" "Establishment of occlusal contact on dental implant" and "Implant materials and biomaterials." The results of these studies are contributed to the Journal of the Japanese Society of Oral Implantology. We are working to enrich the content of the Journal of the Japanese Society of Oral Implantology and aim to expand and promote awarding of "Excellent Thesis Awards" and "Excellent Academia Awards" to the theses

Table 1 Number of specialist and supervisory doctor in oral implantology (September, 2008)

\begin{tabular}{lc}
\hline \multicolumn{1}{c}{ Special qualification of JSOI } & $\begin{array}{c}\text { Number of } \\
\text { doctors }\end{array}$ \\
\hline JSOI certifying doctor of oral implantology & 72 \\
Specialist in oral implantology & 506 \\
Supervisory doctor in oral implantology & 139 \\
\hline
\end{tabular}

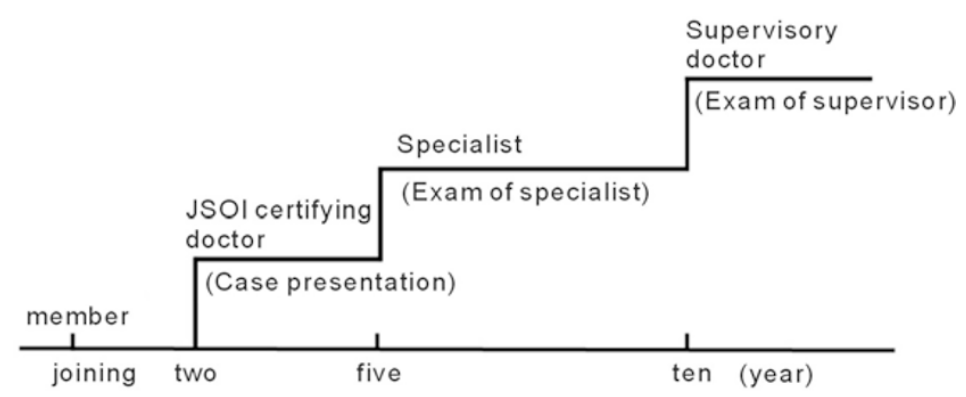

Figure 3 Career path program for specialized qualification 
presented in the Journal of the Japanese Society of Oral Implantology. Further, based on these studies, we are making efforts to establish advanced oral implant treatment in line with specialized treatment guidelines.

The Japanese Society of Oral Implantology organizes annual meetings, including a variety of general, special, and educational lectures as well as symposia, to enhance the knowledge and skills of members. For general lectures and poster presentations, participated in by the members, we will give "Excellent Presentation Awards".

Since 2004, we have selected subjects for the annual meeting: "Advancement in implant treatment-Development into the area of interdisciplinary treatment" for 2004, "Advancement in implant treatment-Seeking evidence and consensus" for 2005, "Advancement in implant treatments-Racial and dietary differences in implant treatments" for 2006, "Specialization in implant treatment-The challenge of raising standards for implants" for 2007, and "Implant treatment in line with the guidelines" for 2008. The main theme for 2009 will be "Safe and reliable implant treatment".

In a highly technical organization like the Japanese Society of Oral Implantology, members' dedication to research and study on advanced implant medical technology will eventually contribute to people and society; however, since we are engaged in medical service, we must fulfill our responsibility and contribute as medical care providers, centered on caring for patients, in the health and medical field, the general medical care world, and the oral health dentistry field.

By accumulating such efforts, the Japanese Society of Oral Implantology and member dentists will become highly regarded and trusted by people and society.

There has been an increasing demand for reliable oral care and implantology, the core of dentistry in the 21 st century. The Japanese Society of Oral Implantology is fully committed to conducting research on oral implantology and advancing implant treatment to meet these public expectations.

Corresponding author: Takayoshi Kawazoe

Chairman of the board of directors, President of Osaka Dental University, President, Japanese Society of Oral Implantology Address: Osaka Dental University, Kuzuha Campus, 8-1, Kuzuhahanazono-cho, Hirakata-shi, Osaka 573-1121, Japan

Tel: $81728643111 \quad$ Fax: $81728643000 \quad$ http://www.osaka-dent.ac.jp 